\section{Successful Treatment of Cervical Ectopic Pregnancy with Multi Dose Methotrexate Therapy}

\section{Abstract}

Cervical ectopic pregnancies account for less than $1 \%$ of all pregnancies. Earlier, it was associated with significant hemorrhage and was treated presumptively with hysterectomy. With the advent of enhanced ultrasound techniques, early detection of these pregnancies has led to the development of more effective conservative management. We present a case of a cervical ectopic pregnancy successfully treated with multi-dose Methotrexate therapy.

A 37-year-old lady, G3P0+2, pregnant for 9 weeks and 4 days, presented with bleeding per vagina, mild lower abdomen and back pain. Serum Beta-hCG done 5 days ago was $950 \mathrm{mIU} / \mathrm{mL}$. She was diagnosed as ectopic cervical pregnancy by clinical examination which was confirmed by transvaginal ultrasonography and subsequently managed by Methotrexate (MTX) Hybrid double dose protocol. Due to rising Beta-hCG and continuous bleeding, it was modified to Multi dose Methotrexate Therapy. Thereafter, the patient was asymptomatic with falling beta-hCG and she was put on a weekly follow up in the clinic.

Keywords: Ectopic pregnancy; Cervical pregnancy; Methrotrexate; Gynaecology

Received: March 31, 2020; Accepted: May 02, 2020; Published: May 06, 2020

\section{Iqbal S ${ }^{1 *}$, lqubal $\mathbf{J}^{2}$, Nowshad $\mathrm{N}^{1}$ and Mohammad K ${ }^{1}$}

1 Department of Obstetrics and Gynecology, Latifa Hospital, Dubai Health Authority Jaddaf, Dubai, UAE

2 Department of Medical Education, Dubai Medical University, Dubai, UAE

*Corresponding author: Iqbal S

Đ suiqbal@dha.gov.ae

Department of Obstetrics and Gynecology, Latifa Hospital, Dubai Health Authority Jaddaf, Dubai, UAE.

Tel: 971569400124

Citation: Iqbal S, Iqbal J, Nowshad N, Mohammad K (2020) Successful Treatment of Cervical Ectopic Pregnancy with Multi Dose Methotrexate Therapy. Gynecol Obstet Case Rep Vol.6 No.2:14

\section{Introduction}

A cervical ectopic pregnancy (CEP) is an ectopic pregnancy, implanted in the uterine endocervix. It is a rare condition with an incidence of less than $0.1 \%$ of all ectopic pregnancies. It is associated with a high morbidity and mortality. The etiology of CEP is not fully understood but reported risk factors for CEP include history of pelvic inflammatory disease, smoking, previous pelvic surgery, previous ectopic pregnancy, intrauterine device use, anatomic anomalies, previous cesarean delivery, previous uterine or cervical surgery, in vitro fertilization and diethylstilbestrol exposure [1].

\section{Case Report}

A 37-year-old lady, G3P0+2 pregnant for 9 weeks and 4 days, conceived by Invitro Fertilization (IVF) presented to the emergency with bleeding per vagina and mild lower abdomen and back pain. This started initially as brownish vaginal discharge and further progressed to frank vaginal bleeding. It soaked half a pad of blood over the span of 2 hours. Beta-hCG which was done 5 days ago showed a reading of $950 \mathrm{mIU} / \mathrm{mL}$.
She has a history of two previous first trimester miscarriages. The first miscarriage was managed by Evacuation of Retained Products of Conception (ERPOC) and second miscarriage was medically managed. The current pregnancy was conceived by IVF- Intracytoplasmic sperm injection (ICSI) and 2 embryos were transferred.

She is a known case of Grave's disease and had radioactive iodine therapy in 2004. Following that, she developed hypothyroidism and is currently on thyroxine $100 \mathrm{mcg}$ for 4 days and $50 \mathrm{mcg}$ for 3 days.

In the emergency, her pain score was $1 / 10$. She was vitally stable with BP of $138 / 84$, Pulse: 85 , Resp: 16 , Temp: $36.7^{\circ} \mathrm{C}, \mathrm{SpO}_{2}: 100 \%$.

On general examination, she was anxious, lying comfortably with no acute distress. Her abdomen was soft and non-tender without masses or organomegaly. The pad was soaked with period-like moderate bleeding and no clots were visible.

Vaginal exam showed no tenderness and closed cervical os. On speculum examination, minimal bleeding was noted along with 
ballooning of the cervix. Bedside transvaginal ultrasound scan shown in Figure 1 elicited no probe tenderness, and revealed an empty uterus with a single gestational sac with a yolk sac measuring $7.4 \mathrm{~mm}$ in the cervical canal. It was corresponding to 5 weeks without a fetal pole and fetal heart. There was evidence of small subchorionic hematoma behind the sac.

The patient was admitted as an ectopic cervical pregnancy and was started on Methotrexate (MTX) Hybrid double dose Protocol with 2 equal doses of MTX $76 \mathrm{mg}\left(50 \mathrm{mg} / \mathrm{m}^{2}\right)$ each to be administered intramuscularly on day 1 and day 4 . Two units of blood were crossmatched and saved. The couple were then explained about the risk of heavy bleeding, uterine perforation, ERPOC, laparoscopy repair, or a Hysterectomy in case of lifethreatening bleeding.

Her Complete Blood Count showed Hemoglobin to be 11.8, MCV 84.9, WBC 9.2, HCT 36.0, and Platelets count of 290,000.

The total Beta-hCG before admission was $950 \mathrm{mIU} / \mathrm{mL}$. The first dose of MTX was given on Day 1and second dose of MTX was administered on day 4. As shown in Figure 2, the value of BetahCG kept rising and the patient was experiencing continuous

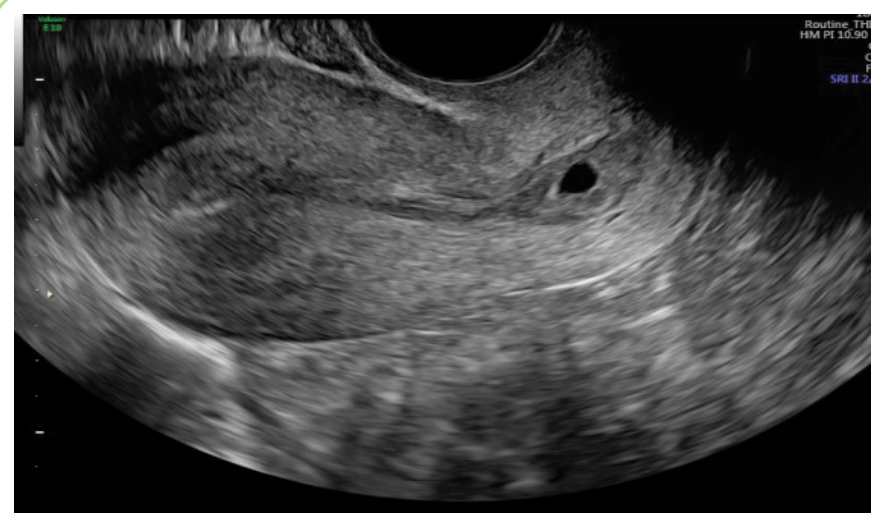

Figure 1 TVUS demonstrates an hourglass shaped uterus with empty uterine cavity, closed internal OS, a gestational and yolk sac corresponding to 5 weeks without a foetal pole and fetal heart, in the cervical canal.

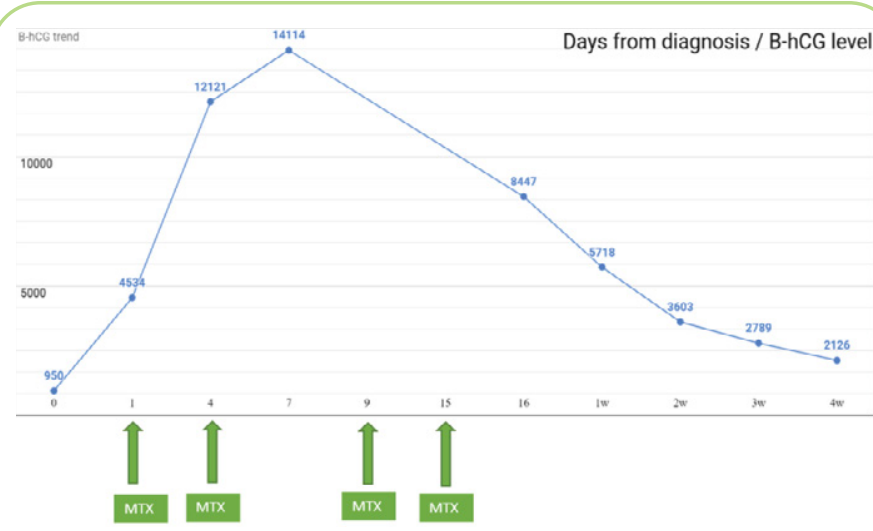

Figure 2 A line graph representing the trend of Beta-hCG levels during multi-dose methotrexate therapy. Arrow indicates the MTX injection. vaginal bleeding. Therefore, it was decided to proceed with Multi-dose MTX therapy and administer 2 more doses of MTX. She received the 3rd dose on Day 9 and 4th dose on Day 15. As she was clinically improving, and Beta-hCG was gradually falling, she was discharged with weekly follow up in clinic for Beta-hCG levels, which is also shown in Figure 2.

\section{Discussion}

The most common symptom of Cervical Ectopic Pregnancy (CEP) is vaginal bleeding, which is often profuse and painless. Lower abdominal pain or cramps occur in less than one-third of the patients, however pain without bleeding is rare. This presentation in early pregnancy should raise concern for CEP. Early diagnosis is critical to avoid severe blood loss and ensure successful treatment. Early CEP can be asymptomatic and is usually found incidentally on ultrasound scan. Unlike tubal ectopic pregnancy where the bleeding is inside the peritoneal cavity, the main risk of cervical pregnancy is uncontrolled vaginal bleeding [2].

Diagnosis can be made on seeing the following features on Ultrasound--an empty uterus, a barrel-shaped cervix, a gestational sac present below the level of the internal cervical OS, the absence of the 'sliding sign' and blood flow around the gestational sac using color Doppler. Early, accurate diagnosis is the key factor in the conservative management of cervical pregnancies. Gestational age less than $12+0$ weeks with absence of fetal cardiac activity and lower serum Beta-hCG levels are associated with more successful conservative management [3].

There are no established criteria for candidates for medical versus surgical treatment as there are for tubal ectopic pregnancy. MTX is first-line therapy for hemodynamically stable women and immediate surgery is required if a patient is unstable. For persistent bleeding after MTX treatment, dilation and endocervical curettage is performed. If these measures fail, the continuous bleeding may be treated with uterine artery embolization (UAE) and then hysterectomy. Multiple injections of intracervical vasopressin can be given around the circumference of the exocervix. Other measures include transvaginal ligation of the cervicovaginal branches of the uterine artery. The suture is placed high just below the lateral vaginal fornix, similar to sutures placed for hemostasis during cold knife conization [2].

A retrospective analysis was done at St George's Hospital, London between April 1997 and September 2004 of all CEPs diagnosed in women attending the early pregnancy unit. CEPs with no visible cardiac activity were advised to use a single dose of methotrexate as first-line management as there appears to be no advantage with multiple-dose regimen in these cases. For CEP with fetal cardiac activity, initial treatment should ideally be local methotrexate or potassium chloride injection with or without interval curettage. If such techniques are not available then multiple-dose systemic methotrexate is an alternative [4].

A case of CEP was reported by Sijanović, Siniša et al. where 
ultrasound guided intra-amniotic methotrexate was unsuccessful and ultimately needed hysteroscopic resection due to severe uncontrolled bleeding [5]. Singh S. reported a case of CEP where cervical tamponade was performed with a Foleys catheter, along with a single-dose intramuscular methotrexate and manual vacuum aspiration. It had a successful outcome [6].

Jaeger et al. reported a case where both cervical and rectal tamponade were used. Intraoperatively a prostaglandin-filled tamponade was placed into the cervical canal. Further profuse bleedings were controlled by the placement of a blocked Foley catheter into the endocervix. A "counter" tamponade of the rectum was performed to ensure sufficient compression and hemostasis. After $24 \mathrm{~h}$ the tamponade was removed [7].

\section{Conclusion}

In conclusion, CEP is extremely rare in occurrence and no definitive treatment guidelines are available. A physician must be competent in managing such cases of ectopic pregnancy on an individual basis, keeping the hemodynamic stability of the patient in mind.

\section{References}

1 Takeda K, Mackay J, Watts S (2018) Successful management of cervical ectopic pregnancy with bilateral uterine artery embolization and methotrexate. Case Rep Emerg Med p. 1-4.

2 Tulandi T (2019) Cervical pregnancy. Up-to-date.

3 Spitzer D, Steiner H, Graf A, Zajc M, Staudach A (1997) Conservative treatment of cervical pregnancy by curettage and local prostaglandin injection. Hum Reprod 12(4): 860-866.

4 Kirk E, Condous G, Haider Z, Syed A, Ojha K, et al. (2006) The

\section{Acknowledgement}

None.

\section{Statement of Ethics}

The patient has given written informed consent to publish this case (including publication of images).

\section{Disclosure Statement}

The authors have no conflicts of interest to declare.

\section{Funding Sources}

None.

\section{Author Contributions}

SI conceived the presented idea of the case report. SI and NN developed the theory. Jl supervised the findings of this work and helped implement the case report. KM contributed to the editing of the manuscript. All authors discussed the results and contributed to the final manuscript.

conservative management of cervical ectopic pregnancies. Ultrasound Obstet Gynecol 27(4): 430-437.

5 Sijanovic S, Vidosavljevic D, Topolovec Z, Milostic-Srb A, Mrcela M (2014) Management of cervical ectopic pregnancy after unsuccessful methotrexate treatment. Iran J Reprod Med 12(4): 285-288.

6 Singh S (2013) Diagnosis and management of cervical ectopic pregnancy. Journal of Human Reproductive Sciences 6(4): 273.

7 Jaeger C, Hauser N, Gallinat R, Kreienberg R, Sauer G, et al. (2006) Cervical ectopic pregnancy: A surgical or medical treatment? Gynecol Surg 4(2): 117-121. 